\title{
Granular-slurry rheology and asphalt compaction
}

\author{
Teng Man ${ }^{1,2}$ and Kimberly Hill 13,4,* \\ ${ }^{1}$ Institute of Advanced Technology, Westlake Institute for Advanced Study, Hangzhou, Zhejiang 310024, China \\ ${ }^{2}$ School of Engineering, Westlake University, Hangzhou, Zhejiang 310024, China \\ ${ }^{3}$ Department of Civil, Environmental and Geo-Engineering University of Minnesota, Minneapolis, MN 55455, United States \\ ${ }^{4}$ St. Anthony Falls Laboratory, University of Minnesota, Minneapolis, MN 55414, United States
}

\begin{abstract}
Hot mixed asphalt (HMA) is a mixture of particles (coarse and fine aggregates) and interstitial fluid (asphalt binder) designed to compact and harden for long-lasting roads. In this study, we implement a two-scale approach to capture the compaction behaviour of hot asphalt mixtures using both a granular-slurry rheology (GSR) at a smaller scale and a discrete element method (DEM) simulation at the scale of a compactor. We show that this modelling effort captures the compaction of HMA with different binder viscosities modified by adding graphene nano-platelets (GNP). This research has the capacity to shed light on how the properties of mixture components can influence compaction efficiency and effectiveness.
\end{abstract}

\section{Introduction to asphalt compaction}

Compaction of granular systems have long been of interest in improving our scientific understanding of dynamics of dry granular materials [1]. Compaction of dry and wet granular materials is also of significant interest for applications from pharmaceutical tablet processing to infrastructure materials installation. In this paper, we focus on such materials widely used in transportation infrastructure: asphalt mixtures.

In the U.S., among more than 2.7 million miles of paved roads, 94\% are paved using compacted asphalt mixtures. When prepared, asphalt mixtures are hot and have the texture of a viscous slurry consisting of particles approximately $d \approx 10 \mathrm{~mm}$ across and finer along with an asphaltene binder with other additives. The initial porosity (i.e., air fraction), often as high as 0.3 needs to be reduced to enhance fracture properties and rutting capacity [2] toward safe, durable and resilient pavement. Typically, engineers compact asphalt in the field by driving heavy rollers over the asphalt pavement, simultaneously shearing and pushing down on the mixture to compact the materials.

Compaction is the most energy-consuming part of asphalt pavement installation. Thus, many experimental efforts aim to investigate how changing inputs to the mixture (e.g., addition of recycled materials) influences compaction efficiency. The "Superpave Gyratory Compactor" (SGC), is one laboratory device used for this purpose and simultaneously shears and applied pressure to its contents (Fig. 1(a)). Figure 1(b) shows measurements from a Brovold SGC for different amounts of graphene nano-platelets (GNP) previously shown to expedite compaction [3].

To understand the manner in which the addition of GNP affects the compaction rate of asphalt mixtures, we

\footnotetext{
* Corresponding author: kmhill@umn.edu
}

A video is available at https://doi.org/10.48448/z6gt-cw54 combine experimental measurements and multi-scale modelling techniques in which we change properties of the mixtures systematically. In this paper, we focus on the effect of the viscosity of the binder / GNP mixture.
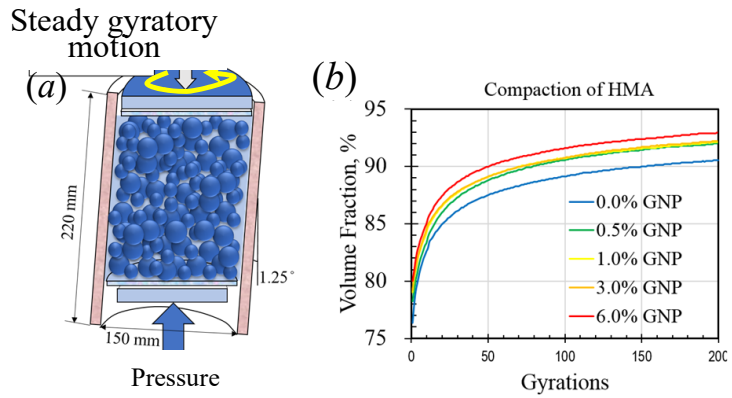

Fig. 1. (a) Schematic dynamics of a SuperPave Gyratory Compactor. (b) Experimental measurements using a Brovold SuperPave Gyratory Compactor showing relationship between number of gyrations and the volume fraction of a hot asphalt mixture (HMA) with different amounts of added graphene nano-platelets (GNP).

\section{Model set-up}

To model our mixture, we consider components commonly prescribed for asphalt roads in Minnesota, United States (where the temperatures have a wide seasonal temperature variation): (1) asphalt binder PG58-28 and (2) particles of grain size distribution shown in Fig. 2, with different quantities of (3) graphene nano-platelets (GNP) of type \#4827.

In the pavement industry, we often distinguish between particles less than and greater than $d \approx$ $2.36 \mathrm{~mm}$. These finer particles, the asphalt binder, and other additives (such as the GNP) are denoted "FAM", 
short for "Fine Aggregate Matrix". We follow this convention here. Specifically, we perform Discrete Element Method (DEM) simulations in which we explicitly represent coarser particles of a grain size distribution between $2.36 \mathrm{~mm}$ and $10 \mathrm{~mm}$ shown in Fig. 2(b), the largest scale. We include the effects of the fine particles (including the GNP) as modifying the properties of the asphalt binder.
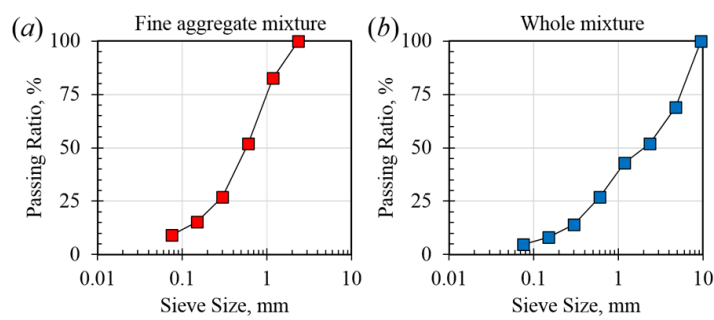

Fig. 2. The grain size distribution of the macroscopic particles our experiments determined by sieving. (a) shows the size distribution of particles $d \leq 2.36 \mathrm{~mm}$, and (b) represents the size distribution of the entire mixture.

\subsection{Coarse-scale modelling}

As is typical in our DEM simulations, the interparticle forces dictated by particle properties come into play only when the particles contact and effectively deform one another. We calculate the normal and tangential forces between particles $i$ and $j$, i.e., $F_{c}^{i j, n}$ (i.e., normal to the plane of contact) and $F_{c}^{i j, t}$ (i.e., tangential to the plane of contact) as follows:

$$
\begin{gathered}
F_{c}^{i j, n}=-k_{n} \delta_{n}^{1.5}-\eta_{n} \delta_{n}^{1.5} \dot{\delta}_{n}, \text { and } \\
F_{t}^{i j, n}=-\min \left(k_{t} \delta_{n}^{0.5} \delta_{t}+\eta_{t} \delta_{n}^{0.25} \dot{\delta}_{t}, \mu_{p} F_{c}^{i j, n}\right) .
\end{gathered}
$$

These contact forces are based on Hertz-Mindlin contact theories [4] with a Coulomb relationship for sliding and damping components based on the derivation outlined by Tsuji et al. [5]. Here, $\delta_{n}$ and $\delta_{t}$ are normal and tangential model deformations (respectively) between particles $i$ and $j$ (Fig. 3(a)). We compute the model coefficients $k_{n}, k_{t}, \eta_{n}, \eta_{n}$, based on material properties similar to that of granite (Table 1) according to established relationships (e.g., Ref. [5]) and use $\mu_{p}=$ 0.1 . based on published experimental measurements (e.g., Ref [6]).

Table 1. Particle material properties used in DEM model.

\begin{tabular}{|c|c|c|}
\hline Density $(\boldsymbol{\rho})$ & $\begin{array}{c}\text { Elastic } \\
\text { modulus }\end{array}$ & $\begin{array}{c}\text { Poisson's } \\
\text { ratio }\end{array}$ \\
\hline $2650 \mathrm{~kg} / \mathrm{m}^{3}$ & $29 \mathrm{GPa}$ & 0.2 \\
\hline
\end{tabular}

We represent the influence of constituents of the FAM in our model via a thick coating on the particles. For neighbouring particles $i$ and $j$ sufficiently close to one another this gives rise to normal and tangential lubrication forces $\left(F_{f}^{i j, n}\right.$ and $F_{f}^{i j, t}$, respectively):

$$
F_{f}^{i j, n}=6 \pi \eta_{1} R_{\mathrm{eff}}^{2} v_{\mathrm{rel}}^{n} / \delta_{g}, \text { and }
$$

$$
\begin{gathered}
F_{f}^{i j, t}=6 \pi \eta_{2} R_{\mathrm{eff}}^{2} v_{\mathrm{rel}}^{t}\left[\frac{8}{15} \ln \left(R_{\mathrm{eff}} / \delta_{g}\right)+0.9588\right], \\
R_{\mathrm{eff}}=\left(2 / d_{i}+2 / d_{j}\right)^{-1}
\end{gathered}
$$

$d_{i}$ and $d_{j}$ are the particle diameters. $\delta_{g}$ is the distance between the nearest surface of the particles (Fig. 3(b)). We assign a maximum and minimum value for $\delta_{g}$ based on the average particle size $\langle d\rangle: \delta_{g, \text { min }}=\langle d\rangle / 10$, which may be thought of as a roughness scale, and $\delta_{g \text {,max }}=\langle d\rangle$, which may be thought of as a coating scale. $v_{\text {rel }}^{n}$ and $v_{\text {rel }}^{t}$ are the relative normal and tangential velocities of the nearest points. $\eta_{1}$ and $\eta_{2}$ are effective fluid viscosities relevant for the normal and tangential directions. Typical derivation of the lubrication theory (Ref. [7-8]) as well as its application usually involves isotropic Newtonian fluid coatings. Yet in this case, our coating - FAM - is a particle-fluid slurry, that is neither isotropic nor Newtonian as we discuss in the next section. Before proceeding, we note that for high shear rates, there may be other fluid forces involved (e.g., Ref. [9]). We suspect these to be minimal based on compaction gyration rates and pressure. Thus, we save these considerations for a future endeavour.
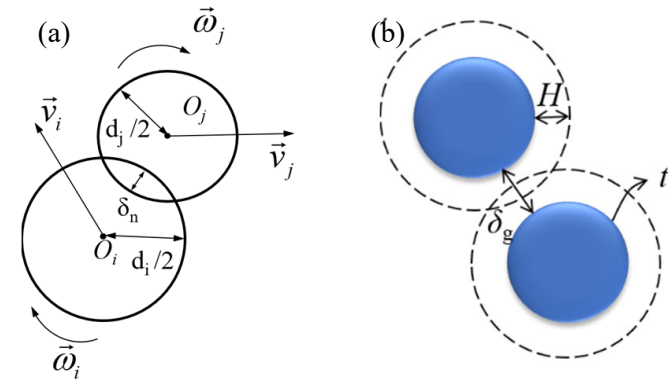

Fig. 3 Sketches showing (a) kinematics relevant to two contacting particles and (b) lubrication force variables.

\subsection{Fine-scale modelling}

For the effective viscosities of the FAM (the asphalt binder mixed with the particles for which $d \leq 2.36 \mathrm{~mm}$ and the GNP), we consider Trulsson et al. (Ref. [9]). They (computationally) sheared $2 \mathrm{~d}$ cylinders in a parallel cell similar to that in Fig. 4, applying a constant normal stress $\sigma$ to roughened boundaries and measuring the necessary shear stress $\tau$ for a given shear rate $\dot{\gamma}$, interstitial fluid viscosity $\eta_{f l}$, particle size and density $\langle d\rangle$ and $\rho$. They proposed a rheology based on both the inertia number $(I)$ and viscous number $(J)$ :

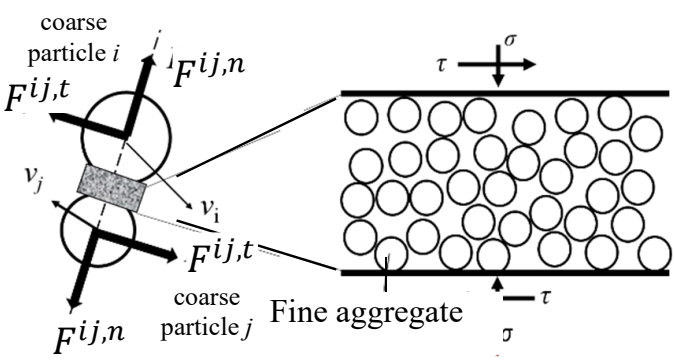

Fig. 4. Sketches illustrating the use of the fine particle-fluid mixture for the viscosities in Eqns. (3)-(4). 


$$
\begin{gathered}
I=\dot{\gamma} d / \sqrt{\rho / \sigma} ; J=\eta_{f} \dot{\gamma} / \sigma, \text { and } \\
K=J+a I^{2} .
\end{gathered}
$$

$\alpha$ is a fitting coefficient. We performed similar $3-d$ simulations of spheres to determine an appropriate rheology of our FAM [10]. We used boundary conditions similar to that in Fig 4, and the interparticle force model described in Section 2.1 (input variables in Tables 1 and 2) with the exception that the fluid viscosities in Eqns. 4 \& 5 are simply the viscosity of the binder + GNP. Fig 5 shows our plots of $\mu_{\text {eff }}=\tau / \sigma$ and solid fraction $\phi$ both vs. $K$ for $a=0.03$. The following relations fit our data well:

$$
\begin{gathered}
\mu_{\mathrm{eff}}=\frac{\tau}{\sigma}=\mu_{1}+\frac{\mu_{2}-\mu_{1}}{1+\sqrt{K_{o} / K}}, \text { and } \\
\phi=\phi_{m} /(1+b \sqrt{K}) .
\end{gathered}
$$

Table 2 provides fit parameters $\mu_{1}, \mu_{2}, K_{O}, \phi_{m}$, and $b$.

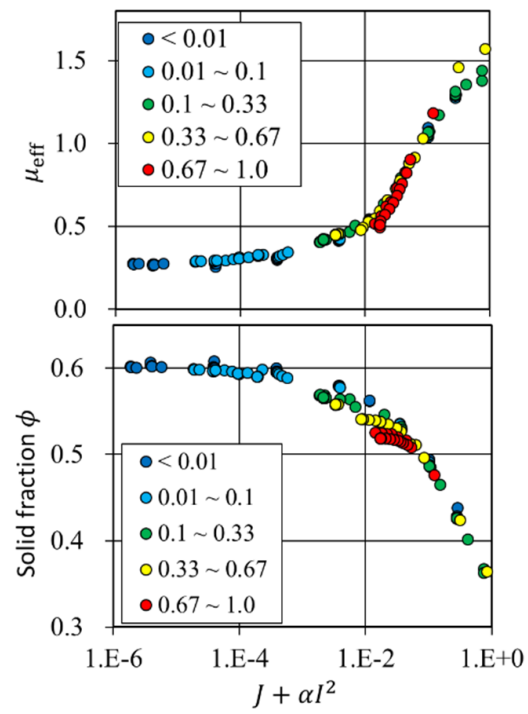

Fig. 5. Data from shear simulations using inputs in Table 2; Legend presents values of $I$ (Eqn. (6)) for the data.

\subsection{Coarse-scale and Fine-scale connection}

We note that we can combine and rewrite the expressions above as effective normal and tangential viscosities $\left(\eta_{1}\right.$ and $\eta_{2}$ in Eqns. $\left.(3,4)\right)$. To do so, we first plot $\mu_{\text {eff }} / K$ vs. $\phi$ (Fig. 6 ) and find the following bestfit relationship $\mu_{\mathrm{eff}} / K=f(\phi)$ :

$$
\mu_{\mathrm{eff}} / K \approx\left(\phi_{m}-\phi\right)^{-5 / 2} / 26 \text {. }
$$

Table 2. Simulation input parameters (along with particle properties in Table 1) and fit parameters for data in Fig. 5.

\begin{tabular}{|c|c|c|c|c|}
\hline $\begin{array}{c}\langle d\rangle \pm \\
\sigma_{d}(\mathrm{~mm})\end{array}$ & $\begin{array}{c}\eta_{1,2}=\eta_{f l} \\
(\mathrm{cP})\end{array}$ & $\begin{array}{c}\sigma \\
(\mathrm{Pa})\end{array}$ & $\begin{array}{c}v_{\text {wall }} \\
(\mathrm{mm} / \mathrm{s})\end{array}$ & $\begin{array}{c}\dot{\gamma} \\
(1 / \mathrm{s})\end{array}$ \\
\hline $1 \frac{1 / 4 \pm 1 / 4}{10^{-4} \text { to }}$ & $\begin{array}{c}100 \text { to } \\
10^{4}\end{array}$ & $\begin{array}{c}10 \text { to } \\
500\end{array}$ & $\begin{array}{c}0.16 \text { to } \\
46\end{array}$ \\
\hline$\mu_{1}(-)$ & $\mu_{2}(-)$ & $K_{o}(-)$ & $\phi_{m}$ & $\mathrm{~b}(-)$ \\
\hline 0.27 & 2.2 & 0.25 & 0.62 & 0.40 \\
\hline
\end{tabular}

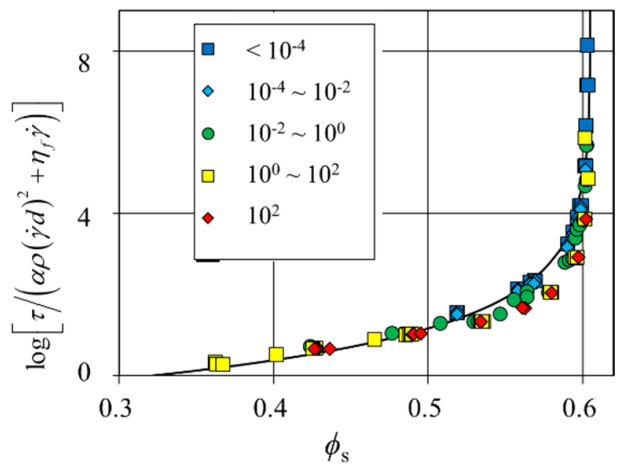

Fig. 6. Parametric plot of measured shear stress values divided by measured normal stress $\left(\mu_{\text {eff }}=\tau / \sigma\right)$ normalized by $K$ (Eqn. 7) plotted vs. measured solid fraction. Legend is $J / I^{2}$. Solid line is best fit power law (Eqn. 10).

Assuming a constant solid fraction in the fine scale lubrication coating on the coarse particles, we write the following relationships for shear and normal stress [11]:

$$
\tau=\eta_{1} \dot{\gamma}, \quad \text { and } \quad \sigma=\eta_{2} \dot{\gamma} .
$$

Here, $\eta_{1}$ and $\eta_{2}$ are the effective viscositites in Eqns. (3-4). We can solve for, $\eta_{1}$ and $\eta_{2}$ using Eqns. (6-11):

$$
\begin{gathered}
\eta_{1}=\tau / \dot{\gamma}=f(\phi)\left(\eta_{f l}+0.03 \rho d^{2} \dot{\gamma}\right), \text { and } \\
\eta_{2}=\sigma / \dot{\gamma}=\eta_{1} b^{2} \phi^{2} / f(\phi)\left(\phi_{m}-\phi\right)^{2} .
\end{gathered}
$$

To incorporate this into the coarse scale simulations, we treat $\dot{\gamma}$ as an effective shear rate between neighboring particles, $v_{\text {rel }}^{t} / \delta_{g} . \rho$ is the particle density in Table $1 . d$ is the average diameter of the finer aggregates $\sim 1 \mathrm{~mm}$. We calculate the coating thicknesss of FAM on the surface of coarse particles based on the volume ratio among binder, fine aggregates and coarse aggregates.

The small size of the graphene nano-platelets (GNP) motivated us to consider them as part of the fluid, effectively modifying $\eta_{f l}$ of the asphalt binder. We measured the temperature and shear-rate dependent viscosities of mixtures of $0.0 \%$ to $6.0 \%$ GNP at temperatures between $100^{\circ} \mathrm{C}$ to $150{ }^{\circ} \mathrm{C}$ (Ref Teng thesis). The behaviors were non-Newtonian (slightly shear thickening) but the effective viscosities based on typical compaction conditions of $\sim 135^{\circ} \mathrm{C}$ and shear rates of $\sim 3$ and $7 \mathrm{~Hz}$ were relatively unchanging [12].

\subsection{Simulation procedure}

We simulated the compaction of asphalt mixture using the boundary conditions of the Brovold SuperPave gyratory compactor. Specifically, we configured a cylindrical shell tilted $1.25^{\circ}$ from vertical as in (Fig. 1a). We placed a horizontal, near-circular plate inside the

Table 3. Weight percent of GNP in experiments with corresponding effective fluid viscosity we measured in the laboratory at $135^{\circ} \mathrm{C}$ and subsequently used in the simulations

\begin{tabular}{|c|c|c|c|c|c|}
\hline $\begin{array}{c}\text { Exp. } \\
\% \mathrm{GNP}\end{array}$ & 0.00 & 0.50 & 1.00 & 3.00 & 6.00 \\
\hline $\begin{array}{c}\mathrm{Sim} . \\
\eta_{f l}(\mathrm{cP})\end{array}$ & 240 & 250 & 270 & 290 & 330 \\
\hline
\end{tabular}


bottom of the shell to apply pressure upon initiation of compaction and to keep material inside. To initiate each compaction experiment, we released a random configuration of our coarse particle mixture of grain size distribution in Fig. 2(b) into the shell. We dropped the particles into the tilted cylinder under the force of gravity and modelled interparticle forces, Eqn's. (1$5,12-13)$. We completed the initiation by placing a horizontal near-circular plate on top of the mixture. We then applied a constant pressure to the bottom plate according to experimental conditions and subjected the top plate to a circular motion slightly off-axis. Throughout the simulation, the circular motion of the top plate moves the system in a gyratory motion and shears the inner mixture. As the bottom plate applies pressure, it also moves vertically, compacting the mix.

\section{Simulation results}

Figure 7 shows the compaction curves of six simulation results plotted with their corresponding experimental results. For the case of $0.0 \%$ of GNP, these results indicate that the "shape" of the compaction curves from our simulations is similar in many ways to those of our experiments with a few differences. At the early compaction stage, the simulated compaction rate was slightly faster than for the real experimental data, while at the late compaction stage, the simulated compaction rate was slower than the physical results. These differences appear common among all our results.

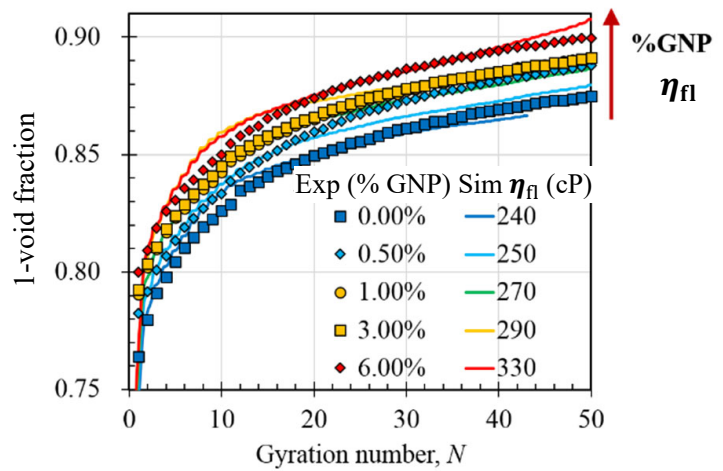

Fig. 7. Experimental and simulation results of the compaction with different amount of GNP additives. The markers represent the experimental results with different percentages of GNP, and the solid curves represent the simulation results with different values of the viscosities of the asphalt binder.

When we add GNP to the asphalt binder, increasing the effective binder viscosity (Table 3 ) compaction proceeds at a higher rate. Similarly when we increase the viscosity of the fluid in our model FAM, our simulated compaction proceeds at a higher rate.

\section{Discussion}

The higher compaction rates for systems in which all things were equal except for the higher fluid viscosities $\boldsymbol{\eta}_{\mathrm{fl}}$ seems somewhat counter-intuitive. However, we note that the apparent increase of efficiency with increasing viscosity is associated with decreased time dependence rather than energy expended by any of these motions. We speculate that the reason for this higher compaction rate may be due to increased lubrication forces. These could effectively reduce interparticle friction allowing particle rearrangements to take place more frequently under applied pressure. A deeper investigation into the dominant interparticle forces would help test this hypothesis.

We conclude by discussing additional similarities and differences among our results. At early times, the simulation compaction rate is higher than analogous experiments. We suspect the sphericity of our simulated particles allows them to slip past one another more readily than the experimental angular particles accountting for this difference. During later times, the experimental slope, i.e., rate of compaction, is similar to that of corresponding simulations. Based on this, we hypothesize that in the later stage viscous forces rather than aggregate shape dominates the mechanics. Finally, a caveat: our work on this problem is relatively limited to date. Additional simulations with investigation of force outputs would help address these issues more completely.

We gratefully acknowledge financial support from the Minnesota Department of Transportation through grant WO20 and from the Center of Transportation Studies at UMN. We thank Mugurel Turos for laboratory assistance, Prof. Marasteaunu for use of equipment in his laboratory, and Prof. Jialiang Le for helpful discussions.

\section{References}

[1] O. Pouliquen, M. Belzons, M. Nicolas, Phys. Rev. Lett. 91, 014301 (2003)

[2] E. Arambula, E. Masad, A. Epps, J. Martin, Mat. Civ. Eng. 19, 655 (2007)

[3] J.L. Le, M.O. Marasteanu, M. Turos, Road Mater. Pavement 21, 1799 (2020)

[4] P.A. Cundall, O.D.L. Strack, Géotechnique 29, 47 (1979)

[5] Y. Tsuji, T. Tanaka, T. Ishida, Powder Technol. 71, 239 (1992)

[6] S.F. Foerster, M.Y. Louge, H. Chang, K. Allia, Phys. Fluids 6, 1108 (1994)

[7] O. Pitois, P. Moucheront, X. Château, J. Colloid Interf Sci 23, 126 (2000)

[8] A.J. Goldman, R.G. Cox, H. Brenner, Chem. Eng. Sci. 22, 637 (1967)

[9] M. Trulsson, B. Andreotti, P. Claudin, Phys. Rev. Lett. 109, 118305 (2012)

[10] T. Man, Q. Feng, K.M. Hill, arXiv preprint, arXiv:1812.07083, (2018)

[11] F. Boyer, E. Guazzelli, O. Pouliquen, Phys. Rev. Lett. 107, 188301 (2011)

[12] T. Man, Rheology of Granular-Fluid Systems and Its Application in the Compaction of Asphalt Mixtures, Ph.D. thesis, Univ. of Minnesota (2019) 